\title{
A study on depression among adolescents with asthma in South Korea using the 15th Korea Youth Risk Behaviour Web-Based Survey
}

\author{
Jin H Park ${ }^{1}$, Mi Jin Kim ${ }^{2}$ \\ ${ }^{1}$ Assistant Professor, Department of Nursing, Changshin University, Changwon; ${ }^{2}$ Assistant Professor, Department of Nursing, Daegu Haany University, \\ Gyeongsan, Korea
}

Purpose: This study investigated the factors that influence depression in adolescents diagnosed with asthma in South Korea, providing basic data supporting efforts to improve adolescents' mental health. Methods: Multiple regression analysis was conducted on 4,020 subjects who had been diagnosed with lifelong asthma among the 57,303 respondents to the 15th Korea Youth Risk Behaviour Web-Based Survey from 2019. Results: The participants were more likely to have depression if they were female, in middle school, their academic achievement was poor, they were drinkers or smokers, if they felt a very high amount of stress, and if they experienced very inadequate recovery from fatigue. Adolescents with asthma were 9.00 times more likely to experience depression when they felt a very high amount of stress $(95 \%$ confidence interval $[\mathrm{CI}]=$ 5.51-14.69, $p<.001)$ than when they felt no stress. Conclusion: Given these factors, measures to improve the mental health of adolescents should be developed and expanded, especially to decrease their stress levels. A separate program that is different from the school's regular health curriculum should be developed to manage the stress levels of adolescents with asthma, such as an after-school program or a program conducted at a local community centre.

Key words: Asthma; Adolescent; Psychological stress; Depression; Smoking

\section{Corresponding author}

Mi Jin Kim

Department of Nursing, Daegu Haany

University, Hanuidae-ro, Gyeongsan

38610, Korea

TEL: +82-53-819-1886

FAX: $+82-53-819-1885$

E-MAIL: mijin77@dhu.ac.kr

Received Jun 1, 2021

Revised Jun 22, 2021

Accepted Jul 9, 2021

\section{INTRO DUCTION}

\section{Background}

Adolescents experience many psychological, physical, and social changes during the transition from childhood to adulthood. These changes manifest in the development of self-consciousness and psychological characteristics that reflect adolescents' desires to escape what they view as an oppressive environment [1]. As adolescents face questions about the meaning and worth of their lives, they also tend to experience inner conflicts about establishing a sense of identity [2]. To overcome the various obstacles adolescents face, it is also important for them to establish values and ethical frameworks to guide their behaviour. This is essential for adolescents to live happy lives and experience healthy growth [3]. Positive thoughts and behaviours experienced during adolescence are closely related to overall life satisfaction, and students who experience positive emotions also tend to have positive selfmotivation and resilience. In addition, self-motivation is closely related to academic performance and participation in regular school activities [4].

A study on the mental health status of teenagers in South Korea found that 1 out of every 2.8 teenagers experienced stress, 1 out of every 4.2 experienced depression, and 1 out of every 8.5 had suicidal thoughts [5]. According to a study on improving and screening the mental health of children and adolescents in Korea, the overall level of psychological wellbeing among Korean children and adolescents has improved over the years, but many still experience poor mental health, and their specific issues are not improving. Therefore, policy efforts are required to maintain and manage the mental health of young people [6]. Mental disorders such as depression and anxiety are the leading cause of disability worldwide among 
people younger than 25 years old. In addition, adolescents aged 15-17 years old comprise almost a quarter of the global population, and managing and screening the mental health of people in this age group is extremely important [7]; thus, mental illness during adolescence, the early identification of problems related to mental health, and mental illness prevention are important topics [8].

Adolescents with chronic physical illness face many obstacles related to their quality of life and behavioural, emotional, educational, and social functions [9]. Furthermore, adolescents who have low life satisfaction have been found to rate their own mental health as poor and present with anxiety and depression [10]. Asthma is among the most common chronic diseases in childhood and adolescence, and it is a major cause of disorders that interfere with adolescents' school activities and daily lives [11]. Furthermore, it has been reported that, in most parts of the world, the prevalence of asthma is likely to increase, particularly among children and adolescents, due to urbanization [12]. The rate of asthma diagnosis among teenagers in Korea was reported to be $8.8 \%$ in 2015, up from 8.5\% in 2007 [13]. Many studies have been conducted in Korea on childhood asthma; a web search using the keywords "child" and "asthma" among Korean sources was found to return 383 manuscripts [11]. Adolescents with allergic diseases such as asthma have been found to have poorer mental health compared to adolescents without allergic diseases, and stress awareness, depression, and suicidal thoughts were 1.15-1.40 times higher among this group [14]. In addition, high degrees of perceived stress, exhaustion, hospital anxiety, depression, and health concerns have been reported among adolescents with asthma [15]. Adolescents with asthma have been found to have more problems regarding their attitudes toward conflict with classmates and teachers in school compared to students without asthma; they are also more likely to be absent from school, in counselling with parents, and in conflict with the people around them [16]. Moreover, they frequently face mental and behavioural problems and are susceptible to other diseases, thus reducing their quality of life and satisfaction, accompanied by stress and depression [17]. Factors related to asthma in adolescents include having a low economic status, being from a single-parent household, and frequently drinking or smoking, and there are also differences according to gender [18]. Another study found that the health behaviour factors related to asthma diagnosis were drinking status, smoking status, physical activity (PA), adolescents' typical nightly amount of sleep, and stress levels [19]. Other studies have also found that adolescents' typical nightly amount of sleep had a significant impact on their stress and likelihood of experiencing depression [20]. Adolescents are future adults who are valuable members of society and mental health during adolescence affects one's entire life into adulthood [8]; therefore, problems that may arise during this period should be recognised early in order to address them. This study used the 15th Korea Youth Risk Behaviour Web-Based Survey (KYRBS) [21] from 2019 to examine the factors that affect depression in adolescents diagnosed with asthma in Korea for the purpose of providing basic data for developing and expanding measures to protect and promote the mental health of teenagers with asthma.

\section{Purpose}

This study aimed to identify the proportion of adolescents with asthma who experience depression and examine the factors that influence their depression. The specific purposes were as follows: 1) to identify the general characteristics, health behaviours (such as drinking status, smoking status, physical education, and PA), and mental health status related to the stress, recovery from fatigue, and depression of the target population, 2) to examine differences in depression related to the general characteristics, health behaviours, and mental health status of the participants, and 3) to determine the factors that affect depression in the target population.

\section{METHODS}

Ethics statement: This study received an Institutional Review Board review exemption (No. X2020016) from Changshin University.

\section{Study Design}

This was a cross-sectional survey in which secondary data analysis was conducted using the 15th KYRBS [21] to identify the effects of depression on adolescents with asthma in Korea.

\section{Participants}

Of the 57,303 people who participated in the 15th KYRBS [21], the 4,020 respondents who answered "yes" to the question "Were you born with or ever diagnosed with asthma?" and who were aged 12 to 18 years were defined as adolescents with asthma.

\section{Data Collection}

The 57,303 people who completed the 15 th KYRBS were intended to provide representative national data. The KYRBS has been conducted annually since 2005 with the aim of un- 
derstanding the current status and trends of health behaviours of teenagers in Korea. The 15th KYRBS [21] was conducted according to the procedures outlined on the website of the Korea Disease Control and Prevention Agency. Starting in April 2019, an online survey was administered to anonymous volunteers across the country who were adolescents from the first year of middle school to the last year of high school.

The sampling process was divided into stages for population stratification, sample allocation, and sample extraction. The number of sample schools was determined by applying the proportional allocation method to match the population composition and sample composition ratio of each stratified variable across 39 school districts of different types (middle schools, general high schools, and specialised high schools) nationwide and divided into 117 layers. For sampling, we used the stratified collection extraction method in which the first extraction unit was the school's permanent random number and the second extraction unit was randomly selected as a class.

The final sample for the 15th KYRBS consisted of a total of 60,100 students across 800 schools (400 middle and 400 high schools). Of the selected sample, 57,303 students from 800 schools ultimately participated in the survey resulting in a 95.3\% participation rate. In the KYRBS, the raw data included logical errors and outliers, and weights were calculated by the Korea Disease Control and Prevention Agency and provided as raw data weighting variables. The weights were multiplied by the weights' posterior correction rate multiplied by the extraction rate reciprocal and response rate reciprocal, and the weights by region, gender, school type (middle, general, specialised school), and grade were calculated to be equal to the number of middle and high school students nationwide as of April 2019.

Responses to the online survey about youth health behaviours were organized using unique numbers that could not be used to determine the respondents' identities; thus, no personal information was disclosed, and anonymity and confidentiality were guaranteed. In addition, the researchers conducted the study after receiving a review exemption from Changshin University, with which the researchers were affiliated (No. X2020016).

\section{Measurements}

The dependent variable in this study was depression among adolescents with asthma, and the independent variables were factors related to depression, including gender, grade, father's education level, mother's education level, economic status, academic achievement, drinking status, smoking status, physical education, PA, stress, and recovery from fatigue.

\section{1) General characteristics}

The variables used to identify the general characteristics of the participants included gender, grade, father's education level, mother's education level, economic status, academic achievement.

Gender was classified as male or female; grade as middle school or high school; father's and mother's education levels as high school or lower, college or above; and economic status and school achievement as high, medium, or low.

\section{2) Health behaviours}

The variables used to examine the health behaviours of the participants included drinking status, smoking status, physical education, and PA. PA included moderate-intensity PA, high-intensity PA, muscular PA, and walking.

Drinking status was classified as "yes" if participants answered "yes" to the question, "have you had more than one drink so far?" and "more than once" to the question, "in the last 30 days, on how many days did you have more than one drink?" If the participants answered "no" for both questions, their drinking status was classified as "no". Smoking status was classified as "yes" if participants answered "yes" to the question, "have you ever taken one or two drags from a cigarette" and "more than once" to the question, "in the last 30 days, on how many days did you smoke at least one cigarette?" If the participants answered "no" for both questions, their smoking status was classified as "no". Physical education was assessed using the question, "have you participated in a physical education class or worked out at a gym within the last 7 days?" The possible answers were "no", "1 day", "2 days", and "3 or more days". Moderate-intensity PA was assessed with the question "in the past 7 days, on how many days did you participate in more than 60 minutes of PA during which your normal heart rate increased?" The possible answers were "none", "1-6 days", and "daily". High-intensity PA was assessed by asking, "in the past seven days, on how many days did you participate in 20 minutes or more of PA that made you short of breath or sweat?" The possible answers consisted of "none", "1-2 days", and "3 or more days". Muscular PA was assessed using the question, "in the past 7 days, on how many days did you participate in muscle-strengthening exercises such as push-ups, sit-ups, lifting weights, lifting dumbbells, using iron bars, or using parallel bars?" Possible answers were "none", "1-2 days", and "3 or more days". Walking was measured using the question "in the past 7 days, on how many days have you walked for more than 60 minutes?" The participants answered "none", "1-6 days", or "daily". Participants who engaged in moderate-intensity PA every day, high-intensity PA on 3 or more days per week, muscular activity on 3 or more days per week, and walked every day were considered to 
practice PA. Participants who answered otherwise were considered not to practice PA.

\section{3) Mental health}

The variables used to assess the mental health of the participants included stress and recovery from fatigue. Stress was measured using the question "how much stress do you usually feel?" Possible answers were "a very high amount", "a high amount", "some", "very little", and "none at all". Recovery from fatigue was assessed using the question "in the past 7 days, do you think the amount of time for which you slept was adequate for recovering from fatigue?" The possible answers were "very adequate", "adequate", "somewhat adequate", "somewhat inadequate", and "very inadequate".

\section{4) Depression}

To assess depression, participants were asked, "have you ever felt so sad or hopeless that you stopped your daily activity for 2 weeks or more within the last 12 months?" The possible responses were "yes" or "no".

\section{Data Analysis}

This study used the 15th KYRBS [21] and, following the analysis guidelines by the Korea Disease Control and Prevention Agency, conducted a composite sample analysis reflecting strata, cluster, weight, and finite population modification using SPSS version 25 (IBM Corp., Armonk, NY, USA). The general characteristics, health behaviours, mental health status, and depression levels of adolescents with asthma were analysed in terms of frequency and percentage. The Rao-Scott $x^{2}$ test was conducted to analyse depression according to the participants' general characteristics, health behaviours, and mental health status. A composite sample logistic regression analysis was conducted to determine the factors that predicted depression in adolescents with asthma.

\section{RESULTS}

\section{General Characteristics, Health Behaviours, and Mental Health Status of Adolescents with Asthma}

In total, $57.2 \%$ of the participants were men, and $42.8 \%$ were women; $47.4 \%$ were middle school students, and $52.6 \%$ were high school students; and $16.7 \%$ of their fathers and $19.6 \%$ of their mothers had a high school or below education level, while $36.0 \%$ and $34.1 \%$, respectively, had a college education or higher. In addition, $45.3 \%$ of participants had a medium economic status, and $40.7 \%$ had high academic achievement. The proportion of participants who responded "no" for drinking status and smoking status was $56.6 \%$ and $84.3 \%$, respectively. For physical education, the highest proportion of respondents (32.9\%) answered "3 or more days a week", followed by "2 days a week"(32.0\%), "1 day a week" (19.3\%), and "none"(15.8\%). PA was practiced by $4.4 \%$ of participants and not practiced by $95.6 \%$, with $60.6 \%$ of participants performing moderate-intensity exercise 1-6 days per week and 34.8\% performing high-intensity PA 1-2 days a week. Finally, 50.4\% of participants reported that they did not perform any muscular exercise at all, and $57.0 \%$ reported that they walked every day.

The highest proportion of participants (37.8\%) responded that they felt some stress, followed by "a high amount" at $29.3 \%$, "a very high amount" at $15.9 \%$, "a little" at $13.4 \%$, and "none at all" at 3.5\%.

In total, $29.9 \%$ of participants reported that their recovery from fatigue was "somewhat adequate", followed by "somewhat inadequate" at 29.6\%, "very inadequate" at 20.3\%, "adequate" at $14.4 \%$, and "very adequate" at $5.8 \%$. The percentage of respondents who did not experience depression was $66.6 \%$, and $33.4 \%$ of respondents did experience depression (Table 1 ).

\section{Differences in Depression According to the General Characteristics, Health Behaviours, and Mental Health Status of Adolescents with Asthma}

The associations between depression among adolescents with asthma and gender (modified $\mathrm{F}=87.21, p<.001$ ), grade (modified $\mathrm{F}=5.68, p=.017$ ), economic status (modified $\mathrm{F}=18.39$, $p<.001$ ), school achievement (modified $\mathrm{F}=18.82, p<.001$ ), drinking status (modified $\mathrm{F}=84.64, p<.001$ ), smoking status (modified $\mathrm{F}=64.64, p<.001$ ), physical education (modified $\mathrm{F}=8.74$, $p<.001$ ), stress (modified $\mathrm{F}=176.11, p<.001$ ), and recovery from fatigue (modified $\mathrm{F}=58.21, p<.001$ ) were found to be significant.

In total, $41.2 \%$ of female respondents reported experiencing depression, which was higher than the proportion of male respondents at $27.5 \%$. In addition, $35.2 \%$ of the participants who experienced depression were high school students, $31.3 \%$ were middle school students, $44.8 \%$ had a low economic status, and $40.5 \%$ had poor achievement in school. The percentage of participants with depression who answered "yes" for drinking status was $42.3 \%$ compared to the $26.5 \%$ of participants who answered that they did not drink. In addition, $47.9 \%$ of smokers experienced depression compared to the $30.6 \%$ of participants who did not smoke.

The percentage of respondents who experienced depression and participated in physical education zero days per week was $40.5 \%$, followed by $34.4 \%$ among those who exercised once per week, $32.4 \%$ among those who exercised twice per week, and $30.2 \%$ among those who exercised three or more 
Table 1. Differences in Depression According to the General Characteristics, Health Behaviours, and Mental Health Status of Adolescents with Asthma $(N=4,020)$

\begin{tabular}{|c|c|c|c|c|c|c|c|}
\hline \multirow{3}{*}{ Characteristics } & \multirow{3}{*}{ Categories } & \multirow{3}{*}{$\mathrm{n}(\%)^{*}$} & \multicolumn{2}{|c|}{ Depression } & \multirow{3}{*}{ Modified $\mathrm{F}^{\dagger}$} & \multirow{3}{*}{$\begin{array}{c}\text { num. df } \\
\text { (denom. df) }\end{array}$} & \multirow{3}{*}{$p$} \\
\hline & & & \multirow{2}{*}{$\begin{array}{c}\text { No } \\
\mathrm{n}\left(\%^{*}\right) \\
\end{array}$} & \multirow{2}{*}{$\begin{array}{c}\text { Yes } \\
\mathrm{n}\left(\%^{*}\right)\end{array}$} & & & \\
\hline & & & & & & & \\
\hline \multirow[t]{2}{*}{ Gender } & Male & $2,300(57.2)$ & $1,668(72.5)$ & $632(27.5)$ & \multirow[t]{2}{*}{87.21} & \multirow{2}{*}{$\begin{array}{c}1.00 \\
(684.00)\end{array}$} & \multirow[t]{2}{*}{$<.001$} \\
\hline & Female & $1,720(42.8)$ & 1,011 (58.8) & 709 (41.2) & & & \\
\hline \multirow[t]{2}{*}{ Grade } & Middle school & $1,905(47.4)$ & $1,308(68.7)$ & $597(31.3)$ & \multirow[t]{2}{*}{5.68} & 1.00 & \multirow[t]{2}{*}{.017} \\
\hline & High school & 2,115 (52.6) & $1,371(64.8)$ & $744(35.2)$ & & $(684.00)$ & \\
\hline \multirow{2}{*}{$\begin{array}{l}\text { Father's education } \\
\text { level }^{\dagger}(n=2,120)\end{array}$} & $\leq$ High school & $671(16.7)$ & $443(66.0)$ & $228(34.0)$ & \multirow[t]{2}{*}{0.14} & 1.00 & \multirow[t]{2}{*}{.708} \\
\hline & $\geq$ College & $1,449(36.0)$ & $972(67.1)$ & $477(32.9)$ & & $(684.00)$ & \\
\hline \multirow{2}{*}{$\begin{array}{l}\text { Mother's education } \\
\text { level }^{\dagger}(n=2,160)\end{array}$} & $\leq$ High school & 787 (19.6) & $520(66.1)$ & 267 (33.9) & 0.06 & 1.00 & .803 \\
\hline & $\geq$ College & 1,373 (34.1) & $912(66.4)$ & 461 (33.6) & & $(684.00)$ & \\
\hline Economic status & High & $1,611(40.1)$ & 1,116 (69.3) & 495 (30.7) & 18.39 & 1.99 & $<.001$ \\
\hline & Medium & $1,820(45.3)$ & 1,238 (68.0) & $582(32.0)$ & & $(1,360.56)$ & \\
\hline & Low & $589(14.6)$ & $325(55.2)$ & $264(44.8)$ & & & \\
\hline Academic & High & $1,635(40.7)$ & 1,154 (70.6) & $481(29.4)$ & 18.82 & 2.00 & $<.001$ \\
\hline achievement & Medium & $1,172(29.1)$ & 803 (68.5) & $369(31.5)$ & & $(1,367.95)$ & \\
\hline & Low & $1,213(30.2)$ & $722(59.5)$ & $491(40.5)$ & & & \\
\hline Drinking status & No & $2,277(56.6)$ & $1,673(73.5)$ & $604(26.5)$ & 84.64 & 1.00 & $<.001$ \\
\hline & Yes & $1,743(43.4)$ & 1,006 (57.7) & 737 (42.3) & & $(684.00)$ & \\
\hline Smoking status & No & $3,390(84.3)$ & 2,351 (69.4) & 1,039 (30.6) & 64.64 & 1.00 & $<.001$ \\
\hline & Yes & 630 (15.7) & $328(52.1)$ & $302(47.9)$ & & $(684.00)$ & \\
\hline Physical education & 0 & $637(15.8)$ & 379 (59.5) & $258(40.5)$ & 8.74 & 2.97 & $<.001$ \\
\hline (days) & 1 & 775 (19.3) & $508(65.6)$ & $267(34.4)$ & & $(2,032.09)$ & \\
\hline & 2 & 1,287 (32.0) & $870(67.6)$ & $417(32.4)$ & & & \\
\hline & $\geq 3$ & 1,321 (32.9) & $922(69.8)$ & $399(30.2)$ & & & \\
\hline PAs & Did not practice & $3,844(95.6)$ & $2,563(66.7)$ & $1,281(33.3)$ & 0.01 & 1.00 & .921 \\
\hline & Practiced & $176(4.4)$ & $116(65.9)$ & $60(34.1)$ & & $(684.00)$ & \\
\hline Moderate-intensity & 0 & 1,293 (32.2) & $883(68.3)$ & $410(31.7)$ & 0.90 & 1.98 & .408 \\
\hline PA (days) & & $2,435(60.6)$ & 1,611 (66.2) & $824(33.8)$ & & $(1,354.08)$ & \\
\hline & Every day & $292(7.2)$ & $185(63.4)$ & $107(36.6)$ & & & \\
\hline Vigorous-intensity & 0 & $1,244(30.9)$ & 817 (65.7) & $427(34.3)$ & 1.57 & 1.97 & .208 \\
\hline PA (days) & $1-2$ & $1,399(34.8)$ & 964 (68.9) & 435 (31.1) & & $(1,344.59)$ & \\
\hline & $\geq 3$ & $1,377(34.3)$ & $898(65.2)$ & $479(34.8)$ & & & \\
\hline Muscular PA (days) & 0 & $2,028(50.4)$ & $1,330(65.6)$ & $698(34.4)$ & 2.99 & 1.97 & .051 \\
\hline & $1-2$ & $1,036(25.8)$ & 722 (69.7) & $314(30.3)$ & & $(1,344.49)$ & \\
\hline & $\geq 3$ & $956(23.8)$ & $627(65.6)$ & $329(34.4)$ & & & \\
\hline Walking (days) & 0 & $201(5.0)$ & $133(66.2)$ & $68(33.8)$ & 0.82 & 1.99 & .439 \\
\hline & $1-6$ & $1,529(38.0)$ & $1,027(67.2)$ & $502(32.8)$ & & $(1,363.88)$ & \\
\hline & Every day & $2,290(57.0)$ & $1,519(66.3)$ & 771 (33.7) & & & \\
\hline Stress & A very high amount & $641(15.9)$ & $206(32.1)$ & $435(67.9)$ & 176.11 & 3.96 & $<.001$ \\
\hline & A high amount & $1,178(29.3)$ & $654(55.5)$ & $524(44.5)$ & & $(2,709.97)$ & \\
\hline & Some & $1,520(37.8)$ & $1,215(79.9)$ & $305(20.1)$ & & & \\
\hline & A little & $539(13.4)$ & $486(90.2)$ & $53(9.8)$ & & & \\
\hline & Not at all & $142(3.5)$ & $118(83.1)$ & $24(16.9)$ & & & \\
\hline Recovery from & Very adequate & $236(5.8)$ & $187(79.2)$ & $49(20.8)$ & 58.21 & 3.97 & $<.001$ \\
\hline fatigue & Adequate & $579(14.4)$ & $464(80.1)$ & $115(19.9)$ & & $(2,711.82)$ & \\
\hline & Somewhat adequate & $1,200(29.9)$ & 878 (73.2) & $322(26.8)$ & & & \\
\hline & Somewhat inadequate & 1,190 (29.6) & $755(63.5)$ & $435(36.5)$ & & & \\
\hline & Very inadequate & 815 (20.3) & $395(48.5)$ & $420(51.5)$ & & & \\
\hline Total & & $4,020(100.0)$ & $2,679(66.6)$ & $1,341(33.4)$ & & & \\
\hline
\end{tabular}

${ }^{*} \mathrm{n}$ is the unweighted frequency and percent $(\%)$ is weighted; ${ }^{\dagger}$ Calculated by complex sample analysis; ${ }^{\dagger}$ Skipped responses were excluded; denom. $\mathrm{df}$, denominator: degrees of freedom; num. df, numerator: degrees of freedom; PA, physical activity. 
times per week. The percentage of respondents who experienced depression among those who reported a very high amount of stress was $67.9 \%$, followed by $44.5 \%$ of respondents who reported a high amount of stress, $20.1 \%$ of respondents who reported some stress, $9.8 \%$ of respondents who reported a little stress, and $16.9 \%$ of respondents who reported no stress. The percentage of participants who experienced depression among those who reported that they had very inadequate recovery from fatigue was $51.5 \%$, followed by $36.5 \%$ of respondents who answered "somewhat inadequate", $26.8 \%$ of respondents who answered "somewhat adequate", and $19.9 \%$ of respondents who answered "adequate" (Table 1).

\section{Factors Influencing Depression in Adolescents with Asthma}

To identify factors affecting depression in adolescents with asthma, we used multiple logistic regression analysis, inputting the significant variables from the Rao-Scott $x^{2}$ test, such as gender, grade, economic status, academic achievement, drinking status, smoking status, physical education, stress, and recovery from fatigue. The resulting model was significant (Wald F=39.11, $p<.001$ ).

The factors that affected depression among adolescents with asthma were gender, grade, academic achievement, drinking status, smoking status, stress, and recovery from fatigue.

The risk of depression was 1.56 times higher for females than for males $(\mathrm{B}=0.44,95 \%$ confidence interval $[\mathrm{CI}]=1.33-1.83$, $p<.001)$, and it was 1.20 times higher for middle school students than for high school students ( $\mathrm{B}=0.18,95 \% \mathrm{CI}=1.00-1.43$, $p=.048)$. The risk of depression was 1.28 times higher when academic achievement was low than when it was high $(B=$ 0.25, 95\% CI=1.06-1.55, $p=.010), 1.49$ times higher when respondents answered "yes" for drinking status than when they answered "no"(B=0.40, 95\% CI=1.26-1.77, $p<.001)$, and 1.54 times higher for smokers than for non-smokers $(B=0.43,95 \%$ $\mathrm{CI}=1.23-1.94, p<.001)$. The risk of depression was also 9.00 times higher when respondents reported a very high amount of stress $(B=2.20,95 \% C I=5.54-14.69, p<.001)$ and 3.71 times higher when respondents reported a high amount of stress $(B=1.31,95 \% C I=2.31-5.97, p<.001)$ than when they reported no stress. Additionally, respondents who reported that they had very inadequate recovery from fatigue were 1.63 times more likely to experience depression than those who reported having very adequate recovery from fatigue $(B=0.49,95 \% \mathrm{CI}=$ 1.08-2.48, $p=.022$ ) (Table 2).

Table 2. Factors Influencing Depression among Adolescents with Asthma $(N=4,020)$

\begin{tabular}{|c|c|c|c|c|c|}
\hline Characteristics & Categories & B & OR & $95 \% \mathrm{CI}$ & $p$ \\
\hline Gender (ref. male) & Female & 0.44 & 1.56 & $1.33-1.83$ & $<.001$ \\
\hline Grade (ref. high school) & Middle school & 0.18 & 1.20 & $1.00-1.43$ & .048 \\
\hline Economic status (ref. high) & $\begin{array}{l}\text { Medium } \\
\text { Low }\end{array}$ & $\begin{array}{r}-0.08 \\
0.20\end{array}$ & $\begin{array}{l}0.93 \\
1.22\end{array}$ & $\begin{array}{l}0.79-1.09 \\
0.96-1.55\end{array}$ & $\begin{array}{l}.348 \\
.105\end{array}$ \\
\hline Academic achievement (ref. high) & $\begin{array}{l}\text { Medium } \\
\text { Low }\end{array}$ & $\begin{array}{r}-0.03 \\
0.25\end{array}$ & $\begin{array}{l}0.97 \\
1.28\end{array}$ & $\begin{array}{l}0.79-1.19 \\
1.06-1.55\end{array}$ & $\begin{array}{l}.752 \\
.010\end{array}$ \\
\hline Drinking status (ref. no) & Yes & 0.40 & 1.49 & $1.26-1.77$ & $<.001$ \\
\hline Smoking status (ref. no) & Yes & 0.43 & 1.54 & $1.23-1.94$ & $<.001$ \\
\hline Physical education (days) (ref. $\geq 3$ ) & $\begin{array}{l}0 \\
1 \\
2\end{array}$ & $\begin{array}{l}0.19 \\
0.03 \\
0.05\end{array}$ & $\begin{array}{l}1.21 \\
1.03 \\
1.05\end{array}$ & $\begin{array}{l}0.95-1.55 \\
0.82-1.30 \\
0.85-1.29\end{array}$ & $\begin{array}{l}.122 \\
.783 \\
.655\end{array}$ \\
\hline Stress (ref. not at all) & $\begin{array}{l}\text { A very high amount } \\
\text { A high amount } \\
\text { Some } \\
\text { A little }\end{array}$ & $\begin{array}{r}2.20 \\
1.31 \\
0.26 \\
-0.36\end{array}$ & $\begin{array}{l}9.00 \\
3.71 \\
1.30 \\
0.70\end{array}$ & $\begin{array}{l}5.51-14.69 \\
2.31-5.97 \\
0.81-2.07 \\
0.41-1.19\end{array}$ & $\begin{array}{r}<.001 \\
<.001 \\
.278 \\
.184\end{array}$ \\
\hline Recovery from fatigue (ref. very adequate) & $\begin{array}{l}\text { Adequate } \\
\text { Somewhat adequate } \\
\text { Somewhat inadequate } \\
\text { Very inadequate }\end{array}$ & $\begin{array}{r}-0.18 \\
0.11 \\
0.34 \\
0.49\end{array}$ & $\begin{array}{l}0.83 \\
1.11 \\
1.40 \\
1.63\end{array}$ & $\begin{array}{l}0.53-1.31 \\
0.73-1.70 \\
0.94-2.09 \\
1.08-2.48\end{array}$ & $\begin{array}{l}.424 \\
.625 \\
.103 \\
.022\end{array}$ \\
\hline & \multicolumn{5}{|c|}{ Nagelkerke $R^{2}=.273$, Cox and Snell $R^{2}=.196$, Wald $F=39.11, p<.001$} \\
\hline
\end{tabular}

CI, confidence interval; OR, odds ratio; ref., reference. 


\section{DISCUSSION}

This study used the 15th KYRBS [21] to determine the factors that affected depression among adolescents with asthma in Korea. It found that adolescents with asthma were more likely to feel depressed when they were female, were in middle school, had poor academic achievement, were drinkers or smokers, felt a very high amount and a high amount of stress, and experienced very inadequate recovery from fatigue. These results also showed that the number of adolescents with asthma who experienced depression was 9 times higher when they reported very high stress levels.

A study by Choi and Jeon [5] found that 1 in every 2.8 adolescents was exposed to stress, 1 in 4.2 adolescents experienced depression, and 1 in 8.5 adolescents experienced suicidal thoughts. In addition, their study found that the mental health status of teenagers who exhibited unhealthy behaviours such as drinking and smoking, as well as those who were in a socially and economically vulnerable environment, tended to be poor, which the results of this study support. Choi and Jeon's study also found that stress among female teenagers was higher than among male teenagers, and the stress levels of adolescents generally increased as their grade levels increased, with $30.3 \%$ of first-year middle school students and $41.4 \%$ of third-year high school students reporting stress [5]. This study's results, however, indicate that asthmatic adolescents tend to experience more depression in middle school than in high school. Since there is a strong relationship between stress and depression in adolescents, further studies are needed to examine the relationship between adolescent stress and depression in greater detail and the factors that influence their stress levels.

Stress experienced by teenagers can lead to mental disorders, such as the habituation of negative thoughts and depression, which can lead to problematic behaviours such as delinquency and crime. Therefore, mental disorders experienced by teenagers require proper management in daily life [19]. In addition, the results of this study showed that the most significant factor related to depression in adolescents with asthma was stress, which is likely due to the fact that teenagers have high stress-recognition rates, and it is necessary to develop and implement interventions to reduce teenagers' stress levels.

In this study, 33.4\% of teenagers with asthma reported that they experienced depression. Furthermore, among adolescents with asthma who experienced depression, $67.9 \%$ reported that they experienced a very high amount of stress. Based on this result alone, there is a clear relationship between stress and depression among adolescents with asthma. According to a study on chronic stress and asthma among ado- lescents, asthmatic adolescents often experience anxiety and depression when they are exposed to stressors, and those who experience anxiety and depression are at an increased risk of experiencing exacerbated asthma symptoms [22]. In a study that analysed data from a 2014 online survey of youth health behaviours in Korea [14], the rate of depression among adolescents with asthma was $32.6 \%$; in other words, adolescents with asthma were 1.38 times more likely to experience depression compared to adolescents without asthma. In addition, the stress-recognition rate of adolescents with asthma was 40.1\%-1.15 times higher than that of asthma-free adolescents $(36.8 \%)$. Thus, it can be assumed that asthmatic adolescents easily recognize stress and experience depression when they feel stress, thus affecting their mood. Therefore, it is important to intervene and teach adolescents how to control their stress.

The results of this study encompass 4,020 adolescents who had been diagnosed with lifelong asthma. Asthma is the most common chronic disease experienced by youths in Korea today [11]. In other countries, such as the United States, asthma remains a significant public health problem that is highly related to environmental circumstances such as exposure to allergens or pollutants. Therefore, it is necessary to approach to the problem holistically and addressing it in homes, schools, and neighbourhoods simultaneously [23].

In this study, those with low academic achievement, female students, middle school students, and students with experiences drinking and smoking had a high probability of experiencing depression among adolescents with asthma. Another study found a significant association between health behaviours such as smoking, PA, and stress and asthma diagnosis among teenagers in Korea [19]. Depression was found to be more likely in females than in males in another study, and in adolescents with asthma with low economic status and academic achievement [14], which supports the results of the study.

This study found that adolescents with asthma were more likely to feel depressed when they were unable to adequately recover from fatigue. Problems such as sleep disorders and chronic fatigue caused by chronic diseases not only reduce adolescents' physical abilities and academic performance but also increase stress [24].

In addition, it has been found that, for teenagers, the typical nightly amount of sleep, anxiety, and depression are all related, and teenagers who get sufficient sleep are happier even when their grades in school are poor. Adolescents with insufficient sleep, however, were more likely to partake in unhealthy behaviours such as smoking and drinking and reported high levels of anxiety, depression, and stress [20]. According to a report on the mental health of adolescents based on their amount of sleep, there is a strong link between one's 
typical amount of sleep and one's stress levels during adolescence [25]. Therefore, it can be inferred that one's typical amount of sleep is shorter in adolescence, and, when recovery from fatigue is inadequate, the stress levels of adolescents increase, in turn affecting depression.

Teenagers with asthma often experience physical and mental problems and have been found to have a strong tendency toward depression and anxiety, with their living environments tending to also affect the prevalence of asthma-related mental problems [26] and their levels of anxiety, perceived stress, and depression [15]. When asthma symptoms are alleviated, the anxiety and depression experienced by adolescents with asthma decrease. However, when asthmatic adolescents' anxiety and depression levels are high, their asthma symptoms also become worse [27].

In addition, although there is still research being conducted to examine the relationship between PA and asthma in adolescents, adolescents who have had asthma for more than 12 months also tended to participate less in PA [28], showing that mental health during adolescence is closely related to physical health. Furthermore, to improve physical health, adolescents must participate in health-promoting behaviours such as receiving sufficient sleep and practicing moderate exercise, thus making it easier for adolescents to manage their mental health [29]. While this prior study did not confirm a direct link between stress and PA related to the exacerbation of asthma in adolescents, improving PA among adolescents, getting enough sleep, and reducing stress and anxiety are considered ways to reduce depression in adolescents with asthma by targeting mediating variables.

According to a comparative study on the mental health of Korean, Chinese, and Japanese teenagers, Korean teenagers tend to be more impulsive and depressed than teenagers in the other two countries. In addition, the likelihood of depression and adopting impulsive behaviours was higher for female students than for male students in all three countries, likely because female adolescents tend to be more aware of other people's views related to their appearance and attributed those views to themselves as part of their self-assessment [30]. Teenagers in all three countries responded that they shared their problems and concerns among peer groups [30], which is considered a healthy method for overcoming adolescent depression by creating an environment in which teenagers can form trustworthy groups of peers with whom to share their problems and concerns.

Asthma in adolescents does not only affect mental health problems such as depression but is also likely to negatively affect behaviour and socialisation as well as exacerbate other mental problems [9]. Adolescents with asthma experience regular worsening of their asthma symptoms, which also neg- atively affects their daily lives, potentially leading to frequently missing classes or reduced PA [6]. Teenagers with chronic diseases also tend to spend a large amount of time at school. Thus, it is important for adolescents with chronic diseases to adapt well to school life and build relationships with peer groups; to this end, it is important for them to establish clear goals and develop a strong sense of identity and resilience [24]. In addition, when adolescents with asthma actively participate in family events, their mental and behavioural problems are reduced [17], which is another healthy method for reducing depression in adolescents with asthma.

The results of previous studies and this study indicate that female adolescents are more aware of stress than male adolescents, that it is difficult for adolescents to manage and control asthma when they partake in unhealthy behaviours such as drinking and smoking, and that inadequate recovery from fatigue negatively affects the mental health of adolescents with asthma. Thus, stress and depression in adolescents with asthma are highly related.

The significance of this study is that it aimed to identify the proportion of adolescents diagnosed with lifelong asthma who experienced depression and the predictive factors that worsened their depression. In this study, the researchers found that, when teenagers with asthma felt very high amounts of stress, their depression also increased substantially. In addition, from the results of this study, it can be concluded that chronic physical illnesses such as asthma affect adolescents' academic performance and mental health. In addition, poor mental health, such as the experience of stress and depression, also relates to adolescents' participation in delinquent behaviours such as drinking and smoking. Adolescents with asthma also reported experiencing inadequate recovery from fatigue, and such feelings of fatigue are closely related to their stress levels. Thus, it is important for adolescents with asthma to manage and be taught how to control their stress levels since their depression tends to worsen when they experience stress. It can also be concluded that depression likely leads adolescents with asthma to participate in unhealthy behaviours such as drinking, smoking, and not getting enough sleep. To put an end to this vicious cycle, local communities and schools must make efforts and work together to provide interventions aimed at adolescents with asthma.

\section{CONCLUSION}

This study was conducted to identify the factors that influenced depression among adolescents diagnosed with asthma in Korea based on data from the 15th KYRBS. This study aimed to provide basic data for the development and expansion of mediating measures designed to improve the men- 
tal health of adolescents with asthma by reducing their depression. The study found that adolescents with asthma who experience depression are more likely to be female, in middle school, have poor academic achievement, practice unhealthy behaviours such as drinking and smoking, experience a very high amount of stress, and recover inadequately from fatigue. Stress was the most significant factor affecting depression in adolescents with asthma. Given these factors, measures to decrease the stress levels and improve the mental health of adolescents with asthma should be designed and put in place, and they should be implemented outside of the context of schools' regular health curriculum. Thus, the researchers suggest implementing after-school programs or programs conducted at local community centres to help manage and improve adolescents' mental health.

This study, however, used large-scale raw data and subjective self-reported responses for assessing stress, recovery from fatigue, and depression. In addition, adolescents in this study were not diagnosed with depression by a physician. Thus, the generalisability of the findings to all adolescents is believed to be limited. Therefore, further studies are needed to examine the factors related to depression in asthmatic adolescents who have been clinically diagnosed with depression and who have visited hospitals for their condition. In addition, future studies should further examine the relationship between stress and depression among adolescents with asthma and the factors that influence their stress levels.

\section{ORCID}

Jin H Park Mi Jin Kim https://orcid.org/0000-0003-1570-5479 https://orcid.org/0000-0003-0208-8176

\section{Authors' contribution}

Conceptualization: all authors; Data curation, Formal analysis: all authors; Writing-original draft, Writing-review and editing: all authors; Final approval of published version: all authors.

\section{Conflict of interest}

No existing or potential conflict of interest relevant to this article was reported.

\section{Funding}

This study was supported by the research fund of Changshin University in 2020.

\section{Data availability}

Please contact the corresponding author for data availability.

\section{Acknowledgements}

None.

\section{REFERENCES}

1. Wilson D, Hockenberry MJ, Rodgers CC. Wong's essential of paediatric nursing. 10th ed. St. Louis, MO: Mosby; 2016. p. 9-90.

2. Negru-Subtirica O, Pop EI, Luyckx K, Dezutter J, Steger MF. The meaningful identity: A longitudinal look at the interplay between identity and meaning in life in adolescence. Developmental Psychology. 2016;52(11):1926-1936. https://doi.org/10.1037/dev0000176

3. Van Beveren ML, Harding K, Beyers W, Braet C. Don't worry, be happy: The role of positive emotionality and adaptive emotion regulation strategies for youth depressive symptoms. British Journal of Clinical Psychology. 2018;57(1):18-41.

https://doi.org/10.1111/bjc.12151

4. Trigueros R, Aguilar-Parra JM, Cangas AJ, Bermejo R, Ferrandiz C, López-Liria R. Influence of emotional intelligence, motivation and resilience on academic performance and the adoption of healthy lifestyle habits among adolescents. International Journal of Environmental Research and Public Health. 2019;16(16):2810.

https://doi.org/10.3390/ijerph16162810

5. Choi JH, Jeon JA. Adolescents' mental health and its relationship with health behaviors. Health and Welfare Policy Forum. 2017; 245:72-83.

6. Kim EK, Dowdy E, Furlong MJ, You S. Mental health profiles and quality of life among Korean adolescents. School Psychology International. 2017;38(1):98-116. https://doi.org/10.1177/0143034316682296

7. Erskine HE, Baxter A, Patton G, Moffitt TE, Patel V, Whiteford HA, et al. The global coverage of prevalence data for mental disorders in children and adolescents. Epidemiology and Psychiatric Sciences. 2017;26(4):395-402.

https://doi.org/10.1017/S2045796015001158

8. Kieling C, Baker-Henningham H, Belfer PM, Conti G, Ertem I, Omigbodun PO, et al. Child and adolescent mental health worldwide: Evidence for action. Lancet. 2011;378(9801):1515-1525. https://doi.org/10.1016/S0140-6736(11)60827-1

9. Bennett S, Shafran R, Coughtrey A, Walker S, Heyman I. Psychological interventions for mental health disorders in children with chronic physical illness: A systematic review. Archives of Disease Childhood. 2015;100(4):308-316.

https://doi.org/10.1136/archdischild-2014-307474

10. Lombardo P, Jones W, Wang L, Shen X, Goldner EM. The funda- 
mental association between mental health and life satisfaction: Results from successive waves of a Canadian national survey. BMC Public Health. 2018;18:1-9. https://doi.org/10.1186/s12889-018-5235-x

11. Kim WK. Studies and proposals of childhood asthma in Korea. Allergy Asthma Respiratory Disease. 2018;6(Suppl 1):52-57. https://doi.org/10.4168/aard.2018.6.S1.S52

12. Lundbäck B, Backman H, Lötvall J, Rönmark E. Is asthma prevalence still increasing? Expert Review of Respiratory Medicine. 2016;10(1):39-51. https://doi.org/10.1586/17476348.2016.1114417

13. Jang B, Kim J, Jang M, Park JH, Kim KS. Relationship between health behaviors, living environment and asthma of adolescents. Journal of Environmental Health Sciences. 2019;45(6):613-621. https://doi.org/10.5668/JEHS.2019.45.6.613

14. Kim J. Mental health in adolescents with allergic diseases-using data from the 2014 Korean Youth's Risk Behavior Web-based Study. Journal of the Korean Society of School Health. 2015;28(2):79-88. https://doi.org/10.15434/kssh.2015.28.2.79

15. Lind N, Nordin M, Palmquist E, Nordin S. Psychological distress in asthma and allergy: The Västerbotten environmental health study. Psychology, Health and Medicine. 2014;19(3):316-323. https://doi.org/10.1080/13548506.2013.806814

16. Blackman JA, Conaway MR. Changes over time in reducing developmental and behavioral comorbidities of asthma in children. Journal of Developmental and Behavioral Pediatrics. 2012;33(1): 24-31. https://doi.org/10.1097/DBP.0b013e3182396895

17. Sweenie R, Basch M, Ding K, Pinto S, Chardon ML, Janicke DM, et al. Subjective social status in adolescents with asthma: Psychosocial and physical health outcomes. Health Psychology. 2020;39(3): 172-178. https://doi.org/10.1037/hea0000822

18. Kim BH, Kim HR. Socioeconomic and sociodemographic factors related to allergic diseases in Korean adolescents based on the 14th Korean Youth Risk Behavior Survey. Journal of the Korea Academia -Industrial cooperation Society. 2019;20(8):494-502. https://doi.org/10.5762/KAIS.2019.20.8.494

19. Park HS, Han JY, Lee NY. The association of health behaviors with stress perception among high school students in Korea: Based on 2015 Korean Youth Risk Behavior Survey. Journal of Korean Academy of Community Health Nursing. 2018;29(1):87-96. https://doi.org/10.12799/jkachn.2018.29.1.87

20. Lee J, Kang J, Rhie S, Chae KY. Impact of sleep duration on emotional status in adolescents. Journal of the Korean Child Neurology Society. 2013;21(3):100-110.
21. Korean Ministry of Education; Ministry of Health and Welfare; Centers for Disease Control and Prevention. The fifteenth Korea Youth Risk Behavior Web-Based Survey statistics. Survey Report. Osong: Korean Center for Disease Control and Prevention; 2016.

22. Landeo-Gutierrez J, Celedón JC. Chronic stress and asthma in adolescents. Annals of Allergy, Asthma an Immunology. 2020;123 (4):393-398. https://doi.org/10.1016/j.anai.2020.07.001

23. Louisias M, Phipatanakul W. Managing asthma in low-income, underrepresented minority, and other disadvantaged pediatric populations: Closing the gap. Current Allergy and Asthma Reports. 2017;17(10):68. https://doi.org/10.1007/s11882-017-0734-x

24. Lee B. Differences in self-perception and school adjustment according to time-use profiles of adolescents with chronic diseases. Korean Journal of Child Studies. 2018;39(4):39-53. https://doi.org/10.5723/kjcs.2018.39.4.39

25. Ki YJ, KimY, Shin WK. Mental health and nutritional intake according to sleep duration in adolescents-Based on the 2007-2016 Korea National Health and Nutrition Examination Survey. Journal of Korean Home Economics Education Association. 2018;30(4): 1-14. https://doi.org/10.19031/jkheea.2018.12.30.4.1

26. Bitsko MJ, Everhart RS, Rubin BK. The adolescent with asthma. Paediatric Respiratory Reviews. 2014;15(2):146-153.

https://doi.org/10.1016/j.prrv.2013.07.003

27. Licari A, Ciprandi R, Marseglia G, Ciprandi G. Anxiety and depression in adolescents with asthma and in their parents: A study in clinical practice. Monaldi Archives for Chest Disease. 2019;89 (3):15-19. https://doi.org/10.4081/monaldi.2019.1063

28. Willeboordse M, Van de Kant Kim KD, Van der Velden CA, Van Schayck CP, Dompeling E. Associations between asthma, overweight and physical activity in children: A cross-sectional study. BMC Public Health. 2016;16(1):1-8. https://doi.org/10.1186/s12889-016-3600-1

29. Adrian M, Charlesworth-Attie S, Vander Stoep A, McCauley E, Becker L. Health promotion behaviors in adolescents: Prevalence and association with mental health status in a statewide sample. Journal of Behavioral Health Services and Research. 2014;41(2): 140-152. https://doi.org/10.1007/s11414-013-9370-y

30. Houri D, Nam EW, Choe EH, Min LZ, Matsumoto K. The mental health of adolescent school children: A comparison among Japan, Korea, and China. Global Health Promotion. 2012;19(3):32-41. https://doi.org/10.1177/1757975912453183 